Research Paper

\title{
Use of green fluorescent protein to monitor Lactobacillus plantarum in the gastrointestinal tract of goats
}

\author{
Xufeng $\operatorname{Han}^{1}$, Lei Wang ${ }^{1}$, Wei $\mathrm{Li}^{1}$, Bibo $\mathrm{Li}^{1}$, Yuxin Yang ${ }^{1}$, Hailong Yan ${ }^{2}$, \\ Lei $\mathrm{Qu}^{2}$, Yulin Chen ${ }^{1}$ \\ ${ }^{1}$ College of Animal Science and Technology, Northwest A\&F University, People's Republic of China, \\ China. \\ ${ }^{2}$ College of Life Science, Yulin University, People's Republic of China, China.
}

Submitted: July 5, 2014; Approved: February 2, 2015.

\begin{abstract}
The experiment aimed to specifically monitor the passage of lactobacilli in vivo after oral administration. The green fluorescent protein (GFP) gene was cloned downstream from the constitutive p32 promoter from L. lactis subsp. cremoris $\mathrm{Wg} 2$. The recombinant expression vector, pLEM415gfp-p32, was electroporated into Lactobacillus plantarum (L. plantarum) isolated from goat. Green fluorescent protein (GFP) was successfully expressed in L. plantarum. After $2 \mathrm{~h}$ post-administration, transformed Lactobacillus could be detectable in all luminal contents. In the rumen, bacteria concentration initially decreased, reached the minimum at $42 \mathrm{~h}$ post-oral administration and then increased. However, this concentration decreased constantly in the duodenum. This result indicated that $L$. plantarum could colonize in the rumen but not in the duodenum.
\end{abstract}

Key words: green fluorescent protein, gastro-intestinal tract, Lactobacillus plantarum, goat.

\section{Introduction}

Lactobacilli are Gram-positive anaerobic or facultative aerobic rods, which can be isolated from human and animal tissues, plants and materials of plant origin, and sewage and fermented products (Coeuret et al., 2003; Lee et al., 2009; Azadnia et al., 2011). Particular lactobacilli strains are considered to present beneficial effects on human and animal health. Therefore, several species of lactobacilli are used as probiotics (Forestier et al., 2001). Lactobacilli are generally regarded as safe (GRAS) by the FDA and are thus regarded as potential vehicles for oral vaccination (Mercenier et al., 1996; Scheppler et al., 2002). Moreover, they can stimulate local cell immunity of intestinal and humoral immunity (Biller et al., 1995; Christensen et al., 2002; Servin, 2004). To provide a beneficial effect on the health of the hosts, the probiotic strain must be able to survive in the gastro-intestinal tract (Fuller, 1992). Therefore, knowing the distribution and movement of lactobacilli in the gastro-intestinal tract, as well as in which tract the strain functions, is necessary. Marking the lactobacilli and tracing them by using green fluorescence protein (GFP) is a good method because GFP presents the advantage of being an auto-fluorescent protein that does not require a substrate. In addition, GFP allows for real-time detection in living cells (Kitts et al., 1995). This method has been applied in monogastric animals but has yet to be reported in ruminants (Yu et al., 2007; Wang et al., 2011).

In the present study, we constructed a constitutive expression vector for lactobacilli by using GFP as the reporter protein. We then electroporated the recombinant into $L$. plantarum, which was isolated from Shanbei White cashmere goats. Finally, the goat was fed with the transformants by using GFP as a visible marker for tracking this strain introduced into the gastrointestinal tract and observing its colonization capability.

\section{Materials and Methods}

\section{Bacteria, plasmid and growth conditions}

Escherichia coli DH5 $\alpha$ was used for the construction and propagation of plasmid and grown in Luria-Bertani

Send correspondence to Y. Chen. College of Animal Science and Technology, Northwest A\&F University, No. 22 Xinong Road, Yangling, 712100 Shaanxi, P.R. China. E-mail: chenyulindk@163.com. 
(LB) medium at $37^{\circ} \mathrm{C}$. Lactobacillus strains were originally isolated from the rumen of goats and were used as recipient strains for genetic construction and grown without shaking in an MRS medium at $37{ }^{\circ} \mathrm{C}$ at static conditions. The bacteria were identified as $L$. plantarum via both phenotypic and genotypic methods (Table 1). When appropriate, antibiotics were added to the culture medium. For $E$. coli and Lactobacillus, ampicillin (Amp) was used at final concentrations of 100 and $50 \mu \mathrm{g} / \mathrm{mL}$, respectively.

In this study, the replicative plasmids, namely, pLEM415, pMG36e, and pEGFP-N1, were used (Table 1). pLEM415 is an E. coli-L. reuteri shuttle vector (Serror et al., 2002) that contained a multi-cloning site and genes for Amp resistance. pMG36e contained the $\mathrm{p} 32$ promoter from L. lactis subsp. cremoris Wg2 (Van de Guchte et al., 1989).

Restriction endonucleases, T4 DNA ligase, and Taq polymerase were purchased from TaKaRa Biotechnology (Dalian, China) and used according to the recommendations of the manufacturers.

\section{PCR amplifications}

The $g f p$ fragment was obtained by PCR amplification from pEGFP-N1, and the primers used were gfpF (5'ATACCGCGGATGGTGAGCAAGGGCGAG-3') and gfpR (5'-GCCGGAGCTCTTACTTGTACAGCTCG TCCATGC-3').The $p 32$ promoter was obtained via PCR amplification from pMG36e, and the primers used were p32F (5'-TGCTCTAGAAATTCGGTCCTCGGGATAT GATAAG-3') and p32R (5'-TCCCCGCGGGAATTTTT CTGCTGAAACGATTGCCAT-3'). Restriction sites added at the 5'-end of each primer are underlined. Agarose gel electrophoresis of plasmid DNA and PCR fragments was performed using the procedure described by Sambrooket et al. (1989). These fragments were recovered via gel extraction by using a DNA Gel-extraction kit (OMEGA, USA).

\section{Construction of expression plasmid carrying the gfp gene}

The PCR products of $g f p$ and $p 32$ were cloned into the pMD19 $\mathrm{T}$ vector and then sequenced. Subsequently, the recombinant pMD19 T-gfp was digested by SacII and SacI and then ligated with SacII-SacI-restricted pLEM415 to construct the recombinant pLEM415-gfp. The recombinant pMD19 T-p32 was then digested by XbaI and SacII and then ligated with $X b a \mathrm{I}-S a c$ II-restricted pLEM415-gfp to construct the recombinant pLEM415-gfp-p32. Thus, the resulting recombinant, pLEM415-gfp-p32, carried the $g f p$ gene under the control of the $p 32$ promoter. The structure of pLEM415-gfp-p32 was verified via restriction analysis, and the $p 32$ and $g f p$ fragment were verified via DNA sequencing.

\section{Electrotransformation procedures}

The pLEM415-gfp-p32 was propagated by transformation into E. coli $\mathrm{DH} 5 \alpha$ competent cells according to manufacturer's instructions. $E$. coli transformants were selected on LB agar plates containing Amp. DH5 $\alpha$ harbouring pLEM415-gfp-p32 was proliferated at $37{ }^{\circ} \mathrm{C}$ for $12 \mathrm{~h}$ with shaking in LB broth containing Amp. pLEM415gfp-p32 was purified from E. coli cultures via the alkaline lysis method (Sambrook et al., 1989). L. plantarum electrocompetent cells were prepared based on a previously described method (Mason et al., 2005). A $50 \mu \mathrm{L}$ concentrated cell suspension was electroporated using a Gene Pulser electroporator (Bio-Rad, Hemel Hempstead, UK) in cuvettes with a $0.2 \mathrm{~cm}$ electrode gap (Flowgen, Ashby de la Zouch, UK) with up to $1 \mu \mathrm{g}$ of plasmid DNA (at a concentration of $100 \mu \mathrm{g} \mathrm{mL}^{-1}$ ). Except where stated, electroporation parameters were $2.0 \mathrm{kV}, 200 \Omega$ parallel resistance, and $25 \mu \mathrm{F}$ capacitance. For phenotypic expression, the cells were diluted immediately into $1 \mathrm{~mL}$ of MRS broth in a $2 \mathrm{~mL}$ vial that was pre-warmed to $37^{\circ} \mathrm{C}$. After $3 \mathrm{~h}$ of incubation, serial dilutions were plated onto MRS agar containing Amp. Plasmids from lactobacilli transformants were

Table 1 - Plasmids and bacterial strains.

\begin{tabular}{|c|c|c|c|}
\hline Plasmids or strain & Relevant characteristics & Antibiotic resistance & Reference or source \\
\hline \multicolumn{4}{|l|}{ Plasmid } \\
\hline pGFP-N1 & plasmid containing gfp gene & $A m p^{r}$ & Jia et al. (2006) \\
\hline pMG36e & E. coli- $L A B$ shuttle vector, containing $\mathrm{P} 32$ promoter & $\mathrm{Em}^{\mathrm{r}}$ & Van de Guchte et al. (1989) \\
\hline pLEM415 & E. coli $-L$. reuteri shuttle vector & $\mathrm{Amp}^{\mathrm{r}} \mathrm{Erm}^{\mathrm{r}}$ & Serror et al. (2002) \\
\hline pLEM415-gfp & pLEM415 containing gfp gene & $A m p^{r} E{ }^{r}$ & This study \\
\hline pLEM415-gfp-p32 & pLEM415-gfp containing p 32 gene & $\mathrm{Amp}^{\mathrm{r}} \mathrm{Erm}^{\mathrm{r}}$ & This study \\
\hline \multicolumn{4}{|l|}{ Bacterial strains } \\
\hline Escherichia coli DH5 $\alpha$ & Transformation host & & Invitrogen \\
\hline Lactobacillus plantarum & Transformation host & & Goat rumen \\
\hline
\end{tabular}

$\mathrm{Em}^{\mathrm{r}}$ : erythromycin resistance; $A \mathrm{mp}^{\mathrm{r}}$ : ampicillin resistance. 
isolated following the method described by Anderson and McKay (1983).

\section{Observation of fluorescence}

For the observation of fluorescent bacteria, lactobacilli cultured overnight under investigation were washed three times in phosphate-buffered saline (PBS, $\mathrm{pH}=7.5$ ) and smeared on microscope slides. Prior to observation, the slides were treated by overlaying with $20 \%$ glycerine. An epifluorescent microscope (AMG) equipped with a GFP filter set (excitation $470 \mathrm{~nm}$; emission $505 \mathrm{~nm}$ to $530 \mathrm{~nm}$ ) was used to visualize fluorescent bacteria. Lactobacilli without pLEM415-gfp-p32 were used as a negative control. To confirm the stability of the replicative plasmid pLEM415-gfp-p32 in lactobacilli, the transformants were tested after culturing continuously for 100 generations at non-selective conditions.

\section{Intestinal distribution of $L$. plantarum-GFP in goats}

To monitor the distribution of L. plantarum-GFP in vivo, eight Shanbei white cashmere goats with fistulas were used in our experiment. Each goat was installed with three fistulas, namely, rumen, duodenum, and ileum fistula. Four goats were orally inoculated with $1 \mathrm{~mL}$ of PBS containing $10^{9}$ cfu of $L$. plantarum harbouring pLEM415-gfp-p32. Other goats were orally inoculated with $1 \mathrm{~mL}$ of PBS as a blank control. After 2, 6, 24, 48, and $72 \mathrm{~h}$, samples from luminal contents in the rumen, duodenum, and ileum were serially diluted in PBS and cultured on MRS plates containing Amp overnight at $37{ }^{\circ} \mathrm{C}$. Bacteria concentrations (cfu $\mathrm{mL}^{-1}$ ) in rumen, duodenum, and ileum were determined according to the number of colonies in MRS plates. The data were analysed by the general linear model procedures of SPSS software (SPSS Inc., Chicago, IL, USA). All experimental procedures with goats used in the present study had been given prior approval by the Experimental Animal Manage Committee of Northwest A\&F University.

\section{Results}

\section{Verification of Recombinant plasmid}

Recombinant plasmid pLEM415-gfp-p32 extracted from DH5a was digested by SacII-SacI and XbaI-SacII, respectively. After enzyme digestion, we performed agarose gel eletrophoresis to identity the DNA fragments. The result of agarose gel eletrophoresis showed that two DNA fragments were about 750 and $200 \mathrm{bp}$, which were consistent with the sizes of $g f p$ and $p 32$ fragment (Data not show). In addition, we also performed agarose gel eletrophoresis to check the PCR products amplified from pLEM415-gfp-P32 by primer $g f p$ and $p 32$, which were about 750 and $200 \mathrm{bp}$ as well. Taken together, these results showed that the expression plasmid of lactobacilli was successfully constructed.

\section{Observation of fluorescence}

For the observation of fluorescent bacteria, transformed bacteria were washed in PBS $(\mathrm{pH}=7.5)$ and observed via direct fluorescence microscopy, and bacteria without pLEM415-gfp-p32 were used as a negative control. The result showed that the transformed bacteria expressed fluorescence, whereas no fluorescence was observed in the negative control (Figure 1). Furthermore, we demonstrated that GFP was detectable in clones of transformed lactobacilli after subculturing for 100 generations without antibiotic. The results suggested that GFP could be stably expressed in the recombinant lactobacilli in vitro.

\section{Monitoring L. plantarum-GFP in the gastrointestinal tract of goat}

After 2, 6, 24, 48, and $72 \mathrm{~h}$ of inoculating goats with the transformant, the geometric means of $\log _{10}$ concentrations of bacteria in rumen, duodenum, and ileum were analysed (Figure 2). At $2 \mathrm{~h}$ post-administration, transformed lactobacilli can be detectable in all luminal contents. In the rumen, the concentrations of bacteria initially decreased, reached minimum at $42 \mathrm{~h}$, and then increased. In the duodenum, however, a constant decrease was observed. Compared with those in other gastrointestinal sections, the transformant colonized in the ileum were highest at any time but without regular changes.

\section{Discussion}

A breakthrough in the transformation of Lactobacillus strains was made when Chassy and Flickinger used electroporation to introduce plasmid and phage DNA into L. casei (Bringel et al., 1989). Since then, methods for the transformation of Lactobacillus have been rapidly developed and widely applied (Lin and Savage, 1986; Bringel and Hubert, 1990; Posno et al., 1991; Bhowmick and Steele, 1993; Mason et al., 2005; Palomino et al., 2010), although few studies have optimized this procedure for lactobacilli. Numerous studies have shown that various parameters, including the competent cell preparation, electrical parameters, and host specificity, can influence the transformation efficiency of Lactobacillus (Hashiba et al., 1990; Wei et al., 1995; Serror et al., 2002).

At present, no standard protocol of competent cell preparation has been established. Numerous studies have shown that culture, suspension, and resuscitation medium of competent cells should be adjusted according to different species of lactic acid bacteria. When competent cells were established according to the procedure described by Mason et al. (2005), the initial transformation frequencies were low (Table 2). Thus, we modified the suspension by adding $0.4 \mathrm{M}$ sucrose and $20 \%$ glycerine, which resulted in improved transformation efficiencies (Table 2). 

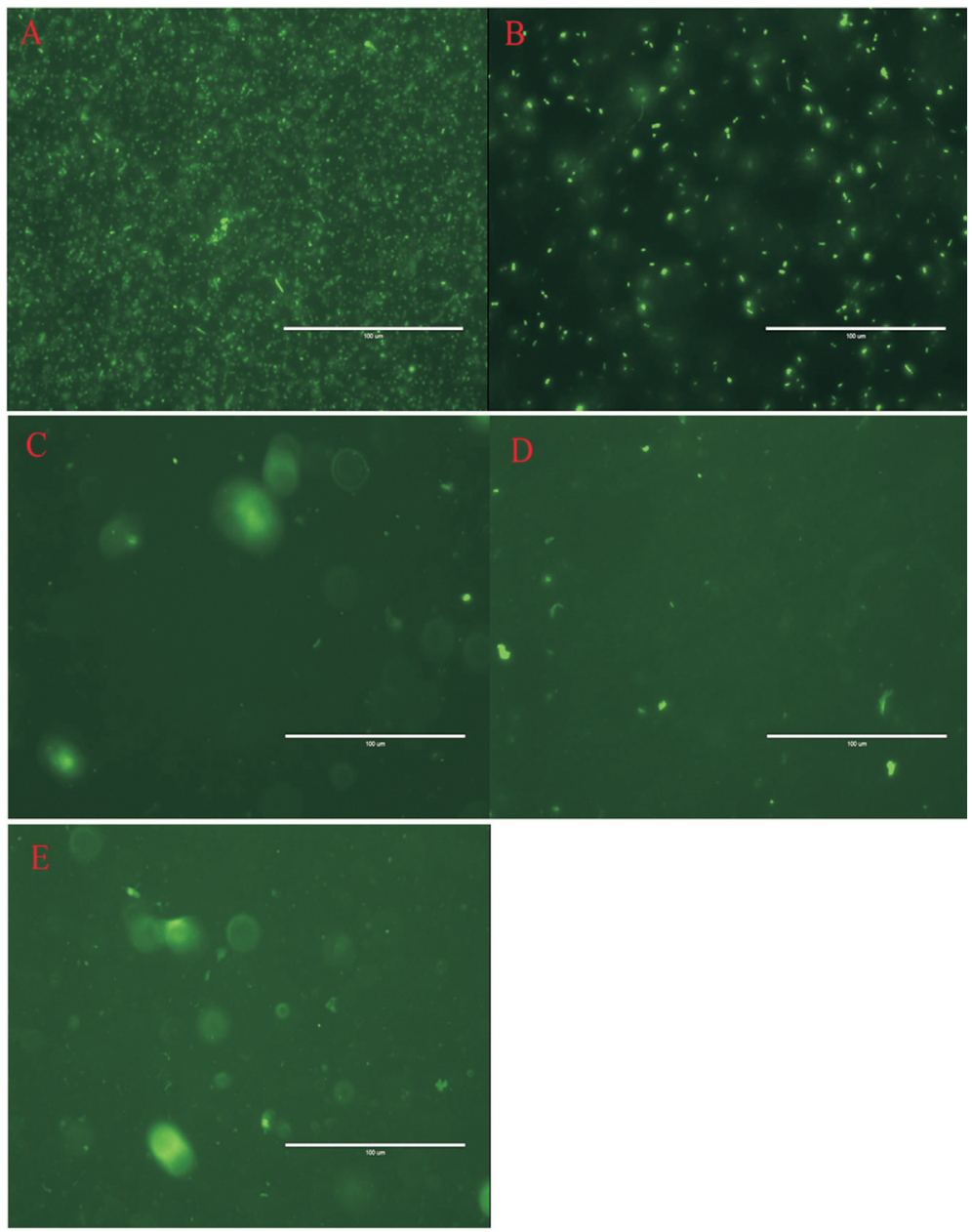

Figure 1 - Fluorescent detection of $L$. plantarum-GFP under fluorescence microscopy. A: fluorescent $E$. coli DH5 $\alpha$ cultured in LB B: L. plantarum-GFP cultured in MRS; C to E: contents of rumen, duodenum, and ileum from goat inoculated with L. plantarum-GFP.

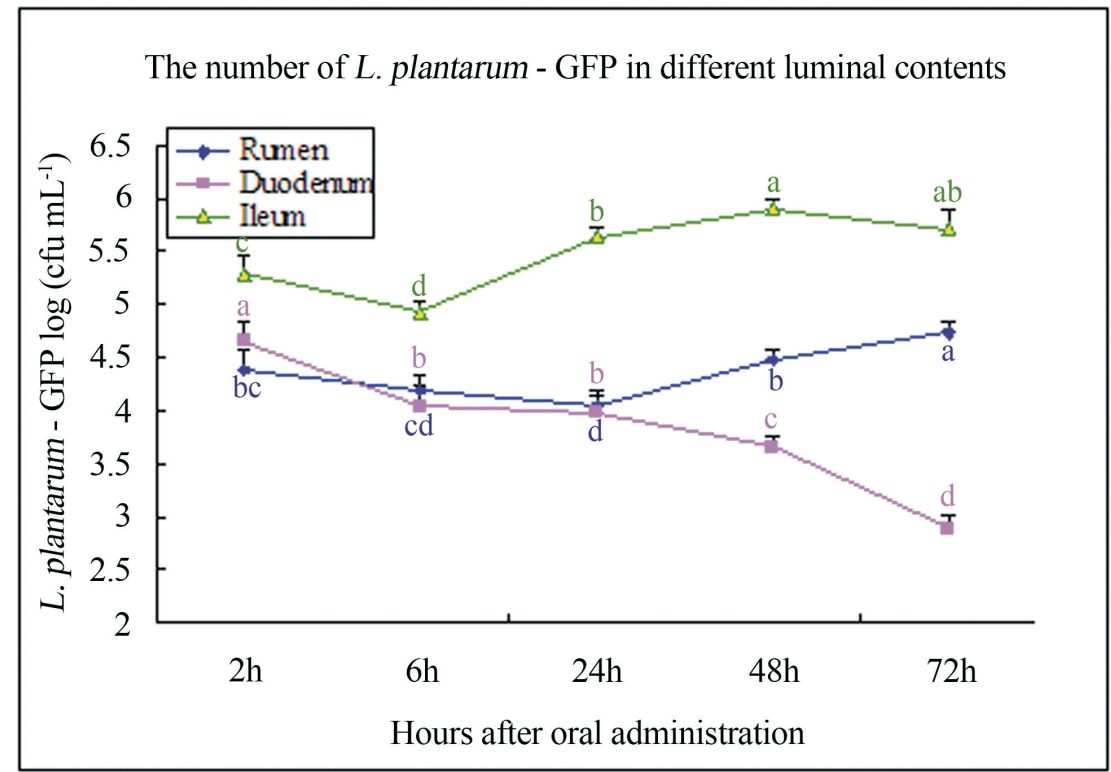

Figure 2 - Trend of the amount of the recombinant strain in the gastrointestinal tract $\left(\log \left(\mathrm{cfu} \mathrm{mL}^{-1}\right)\right)$. Data presented are the means of four goats. 
Table 2 - Transformation frequency of different lactobacilli.

\begin{tabular}{lcc}
\hline Voltage $(\mathrm{kV})$ & Transformation frequency $^{\mathrm{a}}(\mathrm{cfu} / \mu \mathrm{\mu g}$ plasmid DNA $)$ \\
\cline { 2 - 3 } & Mason procedure & Modified procedure \\
\hline 1.5 & $1.1 \times 10^{2}$ & $4.3 \times 10^{3}$ \\
2.0 & $2.7 \times 10^{2}$ & $8.6 \times 10^{3}$ \\
2.5 & Not detected $^{\mathrm{b}}$ & $3.4 \times 10^{3}$ \\
\hline
\end{tabular}

${ }^{a}$ Data are obtained from three experiments.

${ }^{\mathrm{b}}$ Less than 10 transformants per $\mu \mathrm{g}$ plasmid DNA.

Electrical parameters are another important factor that affects electrotransformation efficiency. The optimization of electrical conditions was inconsistent in a previous study (Teresa et al., 2004). Considering the different suspension used, electrical parameters need be optimized to obtain the highest transformation efficiency and a minimum of cell destruction at the same time. In this study, an optimum transformation efficiency was obtained at a pulse strength of $2.0 \mathrm{kV}, 200 \Omega$ parallel resistance, and $25 \mu \mathrm{F}$ capacitance (Table 2).

Gory et al. (2001) demonstrated that the expression of GFP did not alter L. sakei growth and the GFP-L. sakei strains can be monitored both at laboratory growth conditions and in dry sausage samples. Yu et al. (2007) orally inoculated chicken with D17-GFP and found that D17-GFP could propagate and persist at a high level in the gastro-intestinal tract after $2 \mathrm{~h}$. Wang et al. (2011) orally administered GFP-L. lactis $\mathrm{WH}-\mathrm{C} 1$ to mice and found that this Lactococcus strain could exist in all gastro-intestinal tracts for extended periods. The above tests both showed that the recombinant Lactobacillus mainly colonized in the downstream of gastro-intestinal tract. By contrast, trends in the number of Lactobacillus in this study indicated that Lactobacillus could colonize in the rumen but not in the duodenum. Whether lactobacilli could colonize in the ileum requires further studies in the future. Whether exogenous Lactobacillus can adhere in the intestinal mucosa has been considered as one of the selection criteria for probiotic strains (Klaenhamme, 1982; Collins et al., 1998). Thus, our findings indicate that Lactobacillus from rumen can perform an important function in goats as probiotic or expression and delivery vehicles for recombinant proteins.

In addition, the GFP expression of Lactobacillus was successfully applied to animal testing because it offers a simple and rapid method to detect the colonization ability of LAB. This result will aid in better understanding a series of problems, such as the study of relationship between microorganisms and intestinal epithelial cells at the cellular level and the effects on the resident microbiota. In addition, our study will enable us to better understand the distribution and movement of microorganisms in the gastrointestinal tract.

\section{Acknowledgments}

This research was supported by The National Special Research Fund for Non-profit Sector (Agriculture) (20130305905) and China Agriculture Research System (CARS-40-13). We are grateful to Lei Qu and Hailong Yan of Yulin University for their kind help and cooperation during the animal experiments.

\section{References}

Anderson DG, McKay LL (1983) Simple and rapid method for isolating large plasmid DNA from lactic streptococci. Appl Environ Microbiol 46:549-552.

Azadnia P, Zamani MH, Shah AG et al. (2011) Isolation and Identification of Thermophilic Lactobacilli from Traditional Yoghurts of Tribes of Kazerun. J Anim Vet Adv 10:774-776.

Bhowmick T, Steele JL (1993) Development of an electroporation procedure for gene disruption in Lactobacillus helveticus CNRZ 32. J Gen Microbial 139:1433-1439.

Biller JA, Katz AJ, Flores AF et al. (1995) Treatment of recurrent Clostridium difficile colitis with Lactobacillus GG. J Pediatr Gastroenterol Nutr 21:224-226.

Bringel F, Frey L, Hubert JC (1989) Characterization, cloning, curing and distribution in lactic acid bacteria of pLP1, a plasmid from Lactobacillus plantarum CCM 1904 and its use in shuttle vector construction. Plasmid 22:193-202.

Bringel F, Hubert JC (1990) Optimized transformation by electroporation of Lactobacillus plantarum strains with plasmid vectors. Appl Microbial Biotechnol. 33:664-670.

Carmen Collado M, Hernández M (2007) Identification and differentiation of Lactobacillus, Streptococcus and Bifidobacterium species in fermented milk products with bifidobacterium. Microbiol Res 162:86-92.

Christensen HR, Frøkiaer H, Pestka JJ (2002) Lactobacilli differentially modulate expression of cytokines and maturation surface markers in murine dendritic cells. J Immunol 168:171-178.

Coeuret V, Dubernet S, Bernardeau M et al. (2003) Isolation, characterisation and identification of lactobacilli focusing mainly on cheeses and other dairy products. Dairy Science and Technology 83:269-306.

Collins JK, Thornton G, Sullivan GO (1998) Selection of probiotic strains for human application. Int Dairy J 8:487-490.

Forestier C, Champs CD, Vatoux C et al. (2001) Probiotic activities of Lactobacillus casei rhamnosus: in vitro adherence to intestinal cells and antimicrobial properties. Res Microbiol 152:167-173.

Fuller R (1992) Probiotics: The Scientific Basis. Chapmann and Hall, London, pp. 398.

Gory L, Montel MC, Zagorec M (2001) Use of green fluorescent protein to monitor Lactobacillus sakei in fermented meat products. FEMS Microbiol Lett 194:127-133.

Hashiba H, Takiguchi R, Iskii S et al. (1990) Transformation of Lactobacillus helveticus subsp. jugurti with plasmid pLHR by electroporation. Agric Biol Chemistry 54:1537-1541.

Jia Jinghua, Wang Yanping, Zhou Lei et al. (2006) Expression of Pseudomon as aeruginosa Toxin ExoS effectively induces apoptosis in host cells. Infect Immun 74:6557-6570. 
Kitts P, Adams M, Kondepudi A et al. (1995) Green fluorescent protein (GFP): a novel reporter for monitoring gene expression in living cells and organisms. Clontechniques 10:1-3.

Klaenhamme TR (1982) Microbial considerations in selection and preparation of Lactobacillus strains for use as dietary adjuncts. J Dairy Sci 65:1339-1349.

Lee DY, Seo YS, Rayamajhi N et al. (2009) Isolation, characterization, and evaluation of wild isolates of Lactobacillus reuteri from pig feces. The Journal of Microbiology 47:663-672.

Lin JHC, Savage DC (1986) Genetic transformation of rifampicin resistance in Lactobacillus acidophilus. J Gen Microbiol 132:2107-2111.

Mason CK, Collins MA, Thompson K (2005) Modified electroporation protocol for Lactobacilli isolated from the chicken crop facilitates transformation and the use of a genetic tool. J Microbiol Methods 60:353-363.

Mercenier A, Dutot P, Kleinpeter P et al. (1996) Development of lactic acid bacteria as live vectors for oral or local vaccines. Adv Food Sci 18:73-77.

Palomino MM, Allievi MC, Prado-Acosta M et al. (2010) New method for electroporation of Lactobacillus species grown in high salt. J Microbiol Methods 83:164-167.

Posno M, Leer RJ, Van Luijk N et al. (1991) Incompatibility of Lactobacillus vectors with replicons derived from small cryptic Lactobacillus plasmids and segregational instability of the introduced vectors. Appl Environ Microbial 57:1822-1828.
Sambrook J, Fritsch EF, Maniatis T (1989) Molecular Cloning: A Laboratory Manual. 2nd edn. Cold Spring Harbor Laboratory, Cold Spring Harbor, NY.

Scheppler L, Vogel M, Zuercher AW et al. (2002) Recombinant Lactobacillus johnsonii as a mucosal vaccine delivery vehicle. Vaccine 20:2913-2920.

Serror P, Sasaki T, Ehrlich SD et al. (2002) Electrotransformation of Lactobacillus delbrueckii ssp. bulgaricus and $L$. delbrueckiissp. lactis with various plasmids. Appl Environ Microbiol 68:46-52.

Servin AL (2004) Antagonistic activities of lactobacilli and bifidobacteria against microbial pathogens. FEMS Microbio Rev 28:405-440.

Teresa AM, Carmen RM, Mesas JM (2004) Transformation of Lactobacillus plantarum by electroporation with in vitro modified plasmid DNA. FEMS Microbiol Lett 241:73-77.

Van de Guchte M, Van der Vossen JMBM, Kok J et al. (1989) Construction of a lactococcal expression vector: expression of hen egg white lysozyme in Lactococcus lactis subsp. Lactis. Appl Environ Microbiol 55:224-228.

Wang YP, Wang JR, Dai WL (2011) Use of GFP to trace the colonization of Lactococcus lactis WH-C1 in the gastrointestinal tract of mice. J Microbiol Methods 86:390-392.

Wei MQ, Rush CM, Norman HM et al. (1995) An improved method for the transformation of Lactobacillus strains using electroporation. J Microbiol Methods 21:97-109.

Yu QH, Dong SM, Zhu WY et al. (2007) Use of green fluorescent protein to monitor Lactobacillus in the gastro-intestinal tract of chicken. FEMS Microbiol Lett 275:207-213.

Associate Editor: Susana Marta Isay Saad

All the content of the journal, except where otherwise noted, is licensed under a Creative Commons License CC BY-NC. 